\title{
Review: existing epidemiological evidence does not show an association between mumps, measles, and rubella vaccination and autism
}

Wilson K, Mills E, Ross C, et al. Association of autistic spectrum disorder and the measles, mumps, and rubella vaccine: a systematic review of current epidemiological evidence. Arch Pediatr Adolesc Med 2003; 157:628-34.

(1) Are rates of autistic spectrum disorder (ASD) higher in children who have received the measles, mumps, and rubella (MMR) vaccine than in those who have not? (2) Have ASD rates increased as a result of MMR vaccination? (3) Is time of development of ASD associated with time of MMR vaccination? (4) Is a new variant form of ASD associated with MMR vaccination?

\section{METHODS}

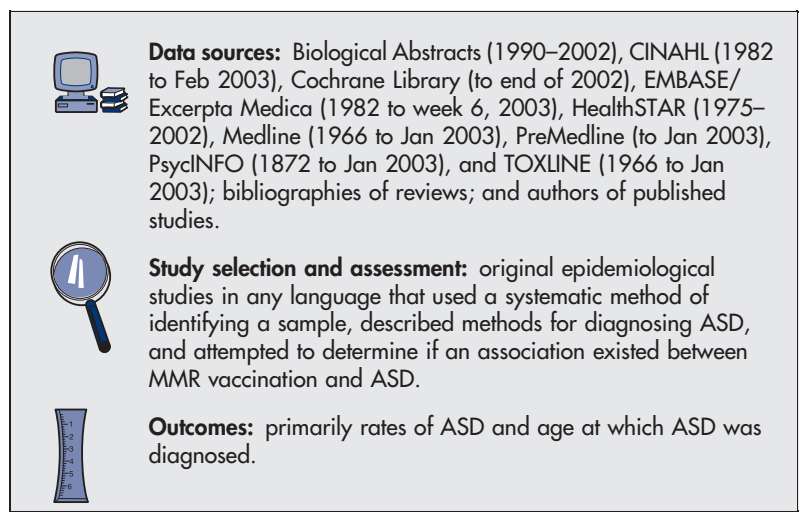

\section{MAIN RESULTS}

12 studies met the selection criteria. (1) Rates of ASD in children who did found no difference between vaccinated and unvaccinated children for ASD (adjusted relative risk [RR] 0.83, 95\% CI 0.65 to 1.07). (2) Changes in ASD rates and changes in MMR vaccination coverage: 6 studies (4 time series, 1 case series, and 1 cross sectional study) found no association between increases in MMR vaccination coverage and ASD rates. (3) Associations between time of MMR vaccination and development of ASD: 3 studies ( 1 cohort study, 1 case series, 1 cross sectional study) found no differences in the mean age of ASD diagnosis (or parental concern) in vaccinated and unvaccinated children. 6 studies ( 2 cohort studies, 3 case series, and 1 cross sectional study) found no increase in ASD diagnosis or features suggestive of ASD after MMR vaccination. (4) Association of variant $A S D$ and $M M R$ vaccination: 4 studies ( 1 cohort study, 1 case series, 1 cross sectional study, and 1 time series) found no association between features of variant ASD and MMR vaccination. and did not have MMR vaccination: 1 cohort study of 537303 children

\section{CONCLUSION}

Existing epidemiological evidence shows that (1) rates of autistic spectrum disorder (ASD) are not higher in children who receive mumps, measles, and rubella (MMR) vaccination; (2) ASD rates have not increased in relation to increased MMR vaccination coverage; (3) time of development of ASD is not associated with MMR vaccination (ie, diagnosis of ASD does not generally occur soon after vaccination); and (4) variant ASD is probably not associated with MMR vaccination, although some of the studies examining this question had important limitations.

\section{Commentary}

1998, Wakefield et al investigated the development of diarrhoea and abdominal pain combined with a loss of acquired skills in 12 paediatric patients. ${ }^{1}$ In 8 cases, parents associated the onset of behavioural symptoms with recent MMR vaccination. Publication of this study, followed by intense media coverage and a pervasive anti-vaccine lobby, heightened parents' fears about MMR vaccine safety and its relation to the development of autism. This is alarming to public health officials who are concerned about decreasing childhood immunisation rates and an increasing risk of measles outbreaks.

The review by Wilson et al adds support to the increasing international body of epidemiological evidence that finds no association between ASD and MMR vaccination. In this area, a hierarchy of evidence exists, with cohort studies providing the strongest evidence to refute the link. Studies that suggest a relation between ASD and MMR vaccination have been small scale case series studies, which provide weak evidence and potentially have significant biases including selection bias.

Immunisation programmes are an important achievement in public health history. Because of their effectiveness, many adults today are unaware of the serious health risks associated with diseases preventable by vaccine. The results of this review are relevant to public health nurses, primary care nurse practitioners, and family medicine physicians involved in the development, promotion, and provision of immunisation programmes. To support parents in their decisions to vaccinate, nurses have a responsibility to be knowledgeable about and educate parents about the evidence-based risks and benefits associated with vaccines, to correct misinformation, and to make clear recommendations.

Susan Jack, RN, PhD School of Nursing, McMaster University Hamilton, Ontario, Canada

For correspondence: Dr K Wilson, Department of Medicine and Health Policy, University of Toronto, Ontario, Canada. kumanan.wilson@uhn.on.ca Source of funding: Canadian Institutes for Health Research. 